\title{
Spectrum Sensing Framework based on Blind Source Separation for Cognitive Radio Environments*
}

\author{
Lina María Sepúlveda Cano** \\ Jhon Jair Quiza Montealegre** \\ Camilo Gil Taborda $a^{* * *}$ \\ Jorge Andrés Gómez ${ }^{* * * *}$
}

\author{
Received: 14/12/2015 - Accepted: 02/05/2016 \\ DOI: $10.22395 /$ rium.v15n29a8
}

\begin{abstract}
The efficient use of spectrum has become an active research area due to its scarcity and underutilization. In a spectrum sharing scenario as cognitive radio (CR), the vacancy of licensed frequency bands could be detected by a secondary user through spectrum sensing techniques. Usually, this sensing approaches are performed with prior knowledge of the channel features. In the present work, a blind spectrum sensing approach based on independent component analysis (ICA) and singular spectrum analysis (SSA) is proposed. The approach is tested and compared with other outcomes. Results show that the proposed scheme is capable of detecting most of the sources with low time consumption, which is a remarkable aspect for online applications with demanding time issues.
\end{abstract}

Key words: spectrum sensing, blind source separation, cognitive radio, ica, ssa

This article is a result from the research" Validation of automatic learning algorithms used in cognitive radio telecommunications systems “, funded by the University of Medellin, during the period 2013-2, 2015-2

** Electronic Engineer. MSc. In Telecommunications Engineering, PhD in Engineering - Automatic. Researcher of the Arkadius Group, University of Medellín. Carrera 87 \# 30-65, Medellín, Colombia. E-mail: 1msepulveda@ udem.edu.co

*** $\quad$ Electronic Engineer. Specialist in Teleinformatics. MSc. In Telecommunications Engineering. Researcher of the Arkadius Group, University of Medellín . Carrera 87 \# 30-65, Medellín, Colombia. E-mail: jhquiza@udem. edu.co

***** Electronic Engineer. MSc. en Multimedia and Communications, UC3M. PhD en Multimedia and Communications, UC3M., Medellín, Colombia. E-mail: cgil@udem.edu.co

****** Electronic Engineer. MSc. In Engineering - Automation. Researcher of the group Bioengineering and Optoelectronics (ByO). Polytechnic University of Madrid . Madrid, España. E-mail: jorge.gomez.garcia@upm.es 


\title{
Método para sensado del espectro basado en separación ciega de fuentes para ambientes de radio cognitiva
}

\begin{abstract}
Resumen
El uso eficiente del espectro se ha convertido en un área de investigación activa, debido a la escasez de este recurso y a su subutilización. En un escenario en el que el espectro es un recurso compartido como en la radio cognitiva (CR), los espacios sin uso dentro de las bandas de frecuencias con licencia podrían ser detectados y posteriormente utilizados por un usuario secundario a través de técnicas de detección y sensado del espectro. Generalmente, estas técnicas de detección se utilizan a partir de un conocimiento previo de las características de canal. En el presente trabajo se propone un enfoque de detección ciega del espectro basado en análisis de componentes independientes (ICA) y análisis de espectro singular (SSA). La técnica de detección se valida a través de simulación, y su desempeño se compara con metodologías propuestas por otros autores en la literatura. Los resultados muestran que el sistema propuesto es capaz de detectar la mayoría de las fuentes con bajo consumo de tiempo, un aspecto que cabe resaltar para aplicaciones en línea con exigencias de tiempo.
\end{abstract}

Palabras clave: sensado del espectro, separación ciega de fuentes, radio cognitiva, ica, ssa. 


\section{INTRODUCTION}

Spectrum sensing is one of the key elements in the cognitive radio (CR) communication, and it is the first step that must be followed before allowing unlicensed users to access vacant licensed channels [1]. In a CR environment, frequency bands that are legally assigned to primary users are exploited by secondary users when primary users are idle [2]; nevertheless, if the primary user needs to establish the communication again, the secondary user has to vacate the channel and seek another one. Detecting the presence of primary signal (spectrum sensing) is a challenging task in CR [3]. Several primary users will perform different modulation schemes, data rates and transmission powers in the presence of both variable propagation environment and interference generated by other secondary users [4]. The spectrum sensing techniques most commonly used are: Energy Detection (ED) [5], Cyclostationary Detection [6] and Matched Filtering [7]. Other techniques proposed to improve the poor performance of ED in noisy environments are the Maximum to Minimum Eigenvalue method (MME) and the Covariance method (COv) [3]. All of these techniques require some previous knowledge that is not always available, regarding the received signals, e.g. the bandwidth, frequency range, modulation scheme, power, among others. Additionally, any sensing spectrum technique should be fast and effective, attempting to reduce processing time and misdetection.

This paper presents a technique for spectrum sensing based on the independent component analysis (ICA) for blind source separation. The proposed scheme includes a single-to-multichannel conversion through singular spectrum analysis (SSA) as an alternative for multi-channelization.

The rest of this paper is organized as follows: first, the spectrum sensing techniques are introduced; then, the proposed scheme for spectrum sensing is described in detail. Lastly, the effectiveness of the proposal is depicted and compared to the other outcomes by using artificial signals with different signal to noise relation (SNR) levels, followed by a discussion of the results and conclusion.

\section{BACKGROUND}

\subsection{Spectrum Sensing}

The spectrum sensing goal in CR is centered in hypothesis (Eq. $(1,2))$ :

$$
\begin{gathered}
H_{0}: x(n)=\eta(n) \\
H_{1}: x(n)=s(n)+\eta(n),
\end{gathered}
$$


where $x(n)$ is the signal received by the secondary user; $s(n)$ is the signal transmitted by the primary user; $\eta(n)$ is an additive white Gaussian noise (AWGN); and $H_{0}$ is the null hypothesis that indicates absence of licensed user signal.

\subsection{Energy Detection (ed)}

In the ED technique, the signal power is measured in a particular frequency band along the time axis. The decision parameter for ED is shown in Eq. (3):

$$
E=\sum_{n=0}^{N}|x(n)|^{2}
$$

where $N$ is the number of samples in the analysis window. In this case, given a threshold $\gamma$, if $E>\gamma$, the hypothesis $H_{1}$ is accepted, and if $E<\gamma$ the hypothesis $H_{0}$ is accepted.

\subsection{Cyclostationary Detection (CD)}

The cyclostationary features are produced by the signal periodicity (or by the periodicity of its statistic measures). The cyclic spectral analysis implies second order transformations of a function and its respective spectral representation [8]. The distinction between primary and secondary users is possible only if different cyclic frequencies are involved [9].

The Spectral Correlation Density (SCD) of a signal could be calculated by Eq. (4) [10]:

$S_{x}^{\alpha}(k)=\mathfrak{F}\left\{C_{x}^{\alpha}(\tau)\right\}=\sum_{\tau=-\infty}^{\infty} C_{x}^{\alpha}(\tau) e^{-j 2 \pi k \tau} \in \mathbb{C}$,

where $\mathfrak{F}\{$.$\} is the Fourier Transform; \alpha$ is the cyclic frequency and $C_{x}^{\alpha}(\tau)$ is the Cyclic Autocorrelation Function (CAF) of $x$, defined in Eq. (5):

$C_{x}^{\alpha}(\tau)=\mathcal{E}\left\{x(n+\tau) x^{*}(n-\tau) e^{j 2 \pi \alpha n}\right\} \in \mathbb{C}$,

where $x^{*}$ is the conjugate version of $x$. The CAF exhibits peaks when the cyclic frequency $\alpha$ has the same value of the fundamental frequencies of the transmitted signal [6].

The frequency-smoothing method is used to estimate the SCD as recommended in [11]: 


$$
\hat{S}_{x}^{\alpha}(l)=\frac{1}{L(N-1) T_{s}} \sum_{v=\frac{-(L-1)}{2}}^{\frac{(L-1)}{2}} X\left(l+\frac{\alpha}{2 F_{s}}+v\right) X^{*}\left(l-\frac{\alpha}{2 F_{s}}+v\right) W(v),
$$

where:

$$
X(v)=\sum_{k=0}^{N-1} x(k) e^{\frac{-j 2 \pi v k}{N}}
$$

where $N$ is the number of samples in the analysis window; $T_{s}$ is the time-sampling increment; $F_{s}$ is the frequency-sampling increment; and $W(v)$ is the frequency smoothing function centered at $v=0$ of width $L$.

\section{PROPOSED SCHEME}

\subsection{Blind Source Separation}

Blind source separation techniques allow separating different signals without previous knowledge about the signal features. The most common technique for source separation is ICA.

In ICA, the received signal is assumed as a linear mixture of statistically independent sources that could be separated maximizing the non-Gaussianity. This technique requires a MIMO system with $p$ sources and $m$ receptors $(p \leq m)$, their combined outputs build the matrix of observable data $\boldsymbol{X} \in \mathbb{R}^{m \times N}, \boldsymbol{X}=\left[x_{i}(1) x_{i}(2) \cdots x_{i}(N)\right]$, where $x_{i}(j)$ is the $j$-th sample (or time instant) of the signal in the $i$-th receptor, and $N$ is the number of samples in the analysis window. The principal idea in ICA, is to get the original sources $\boldsymbol{S}$ from the matrix of observable data $\boldsymbol{X}$ as in Eq. (8):

$$
X=A S,
$$

where $\boldsymbol{A} \in \mathbb{R}^{p \times N}$ is the mixture matrix and $\boldsymbol{S}=\left[s_{l}(1) s_{l}(2) \cdots s_{l}(\mathrm{n})\right]$, where $s_{l}(j)$ is the $j$-th sample (or time instant) of the signal transmitted by the $l$-th source, and $N$ is the number of samples in the analysis window.

If the matrix $\boldsymbol{A}$ is known, the source data could be reconstructed by its pseudoinverse matrix. If $\boldsymbol{A}$ is unknown, it is necessary to calculate $\boldsymbol{A}$ and $\boldsymbol{S}$ from the observation matrix $\boldsymbol{X}$ as in Eq: (9), in such a way that the estimated components $\hat{\boldsymbol{S}}$ must be the linear combination of the data with the maximum independence [12].

$$
\hat{\boldsymbol{S}}=\boldsymbol{W} \boldsymbol{X},
$$


where $\boldsymbol{W} \in \mathbb{R}^{p \times m} \quad$ is a matrix that maximizes the statistical independence.

\subsection{MS - ICA}

In this work, the algorithm proposed by Molgedey [13], MS - ICA, is chosen (Algorithm 1 [14]) due to its performance in online applications [15].

\section{Algorithm 1. MS - ICA}

Input: Data matrix $\boldsymbol{X}$

Output: Estimated source matrix $\hat{\boldsymbol{S}}$

1. Center the observation matrix variance $\tilde{\boldsymbol{X}}=\boldsymbol{X}-\mathcal{E}\{\boldsymbol{X}\}$, where $\mathcal{E}\{$.$\} is the$ expectation operator.

2. Whitening: transform $\boldsymbol{X}$ with non correlated components and unit variance

a) Calculate the covariance matrix $\operatorname{COV}\{\tilde{\boldsymbol{X}}\}=\boldsymbol{R}_{\tilde{\boldsymbol{X}}}=\tilde{\boldsymbol{X}} \tilde{\boldsymbol{X}}^{\top}$

b) Find the eigenvalues $\boldsymbol{R}_{\tilde{\boldsymbol{X}}}=\boldsymbol{V} \mathbf{O} \boldsymbol{V}^{\top}$, where $\boldsymbol{V}$ is the eigenvector matrix and $\mathbf{O}$ is a diagonal matrix of eigenvalues

c) Find the transformation matrix $\boldsymbol{P}=\boldsymbol{V} \boldsymbol{O}^{-\frac{1}{2}} \boldsymbol{V}^{\top}$

d) Calculate the data matrix projection $\boldsymbol{Y}=\boldsymbol{P} \tilde{\boldsymbol{X}}$

3. Fix the lag $\tau$

4. Solve the eigenvalue problem for the ratio matrix

$\boldsymbol{Q}=\boldsymbol{Y}_{\tau} \boldsymbol{Y}^{\top}\left(\boldsymbol{Y} \boldsymbol{Y}^{\top}\right)^{-1} \approx \boldsymbol{A} \boldsymbol{C}_{\boldsymbol{S}}(\tau) \boldsymbol{C}_{\boldsymbol{S}}(0) \boldsymbol{A}^{-1}$, where $\boldsymbol{C}_{\boldsymbol{S}}(\tau)$ is the autocorrelation matrix of $\boldsymbol{S}$ at lag $\tau$

5. Compute the matrix $\boldsymbol{A}$ as the eigenvalues matrix of $\boldsymbol{Q}$ [16].

6. Find the mixture matrix $\boldsymbol{W}=\boldsymbol{A}^{-1}$

7. Calculate the source matrix $\hat{\boldsymbol{S}}$ by Eq. (9)

The lag parameter $\tau$ could be calculated as proposed in [16]: a) compute $\hat{\boldsymbol{S}}$ as in algorithm 1 for $\tau=1$; b) find the minimum value of $\delta(\tau)$ in Eq. (10) by iterating the parameter $\tau$, where $c_{S_{i}}$ are the normalized autocorrelation functions; and c) recalculate $\hat{\boldsymbol{S}}$ after $q$ iterations. 


$$
\delta(\tau)=\sum_{i=0}^{q-1}\left|c_{S_{i+1}}(\tau)-c_{S_{i}}(\tau)-\frac{1}{q-1}\right|
$$

\subsection{Single-to-Multichannel Conversion}

Aiming to find a matrix representation for a single-channel data, the procedure proposed by [17] through SSA was applied as follows:

1. Calculate the autocorrelation function of $x(n)$ as in Eq. (11), where $l=0, \ldots, L-1$ and $L$ is the analysis window length. A suitable value for $L$ is an important issue in SSA. In this work, the value is fixed as one more than the number of peaks in the periodogram of the received signal $x(n)$, aiming to find an approximate number of expected sources.

$$
\boldsymbol{C}_{x}(l)=\sum_{i=1}^{N-L+1}\left(x_{i+l}-\mathcal{E}\{x\}\right)\left(x_{i}-\mathcal{E}\{x\}\right)
$$

2. Find the Toeplitz matrix $\boldsymbol{T}_{o}$ of the autocorrelation function.

3. Calculate de eigendecomposition of $\boldsymbol{T}_{o}$ as $\boldsymbol{T}_{o}=\boldsymbol{V} \mathbf{O} \boldsymbol{V}^{\top}$. Sort eigenvalues (O) in descending order and preserve the most informative components.

4. Create the Hankel matrix $\boldsymbol{T}_{H}$ of $x(n)$, with $K=N-L+1$ (Eq. (12)).

$$
\boldsymbol{T}_{H}=\left[\begin{array}{ccc}
x_{1} & \cdots & x_{1+L-1} \\
\vdots & \ddots & \vdots \\
x_{K} & \cdots & x_{K+L-1}
\end{array}\right]
$$

5. Project $\boldsymbol{T}_{H}$ into the subspace of eigenvectors of $\boldsymbol{T}_{o}$, i.e., $\boldsymbol{Y}=\boldsymbol{T}_{H} \boldsymbol{V}$ or $\boldsymbol{Y}_{j}=\boldsymbol{V}_{j} \boldsymbol{V}_{j}^{\top} \boldsymbol{T}_{H}^{\top}$ The main idea is to define the temporal behaviors.

6. Reconstruct $\boldsymbol{X}$ as a sum of the projections obtained by the inverse Hankel procedure of $\boldsymbol{Y}_{j}$.

7. Calculate algorithm 1 with the following modifications:

a. Choose two subsets, i.e., $j=2$.

b. Use the first subset as the input of the algorithm.

c. Divide the remaining subset into two subsets.

d. Go to step b. and repeat the process. 


\section{EXPERIMENTAL SET-UP}

The different schemes for spectrum sensing are tested with an artificial mixture signal defined by Eq. (13).

$$
x(n)=\sum_{i} a_{i} \cos \left(2 \pi f_{i} n+\phi_{i}\right)+\eta(n)
$$

The length $N$ of $x(n)$ with $n=1, \ldots, N$ is fixed in 4,000 bins, the sampling frequency $f_{s}=10 \mathrm{kHz}$, and the parameter $L=512$ for the cyclostationary analysis (smoothed window length). The white Gaussian noise $\eta(n)$ is a variable parameter, with SNR between -10 and $20 \mathrm{~dB}$.

In order to check the technique's efficiency, some variations in the number of original sources and noise power $\left(P_{\eta}\right)$ are made. The amplitude of the sources is $a_{i}=1$ and the phase $\phi_{i}=0$. For ED, a system with six FIR filters is implemented, each one with order 100, Hamming window, bandwidth $520 \mathrm{~Hz}$, a center frequency of $f_{i}$ and $10 \mathrm{~Hz}$ of overlapping; then, the procedure recommended by [18] is followed for each channel with 1,000 Monte Carlo simulations, aiming to determine whether a channel is available or not. For $\mathrm{CD}$, the parameter $\mu$ is fixed by heuristic tuning and it determines when a peak of the CAF corresponds to a fundamental frequency or not.

The elapsed time that the algorithms took to run is measured in an Intel ${ }^{\circledR} \mathrm{Core}^{\mathrm{TM}}$ i5-2,400 with 4 GB of RAM memory and Python ${ }^{\circledR}$ of 64 bits.

\section{RESULTS AND DISCUSSION}

Table 1 summarizes the results for the three tested schemes. The technique most sensitive to noise is ED, with the disadvantage that if the frequency bands are unknown, the algorithm is unable of detect different frequency components. The mean time elapsed for this technique is $97.85 \pm 1.02 \mathrm{~s}$, and tends to increase with the number of channels. The CD and MS-ICA's are noise-robust algorithms. The time elapsed for CD is around $106.94 \pm 1.77 \mathrm{~s}$ that could be compensated by its precision. The CD sensing technique generates some false positives, but the rate of false negatives is zero. If the intention of the secondary user is to search for vacancy without disturbing the primary user, and the time is not a critical issue in the application, this technique could be a convenient solution. The only inconvenient detected is the parameter $\mu$, because the algorithm performance depends on the choice of this parameter. 
Table 1. Spectrum sensing test results

\begin{tabular}{|c|c|c|c|c|}
\hline Technique & $\begin{array}{l}\text { Sources } \\
\text { (Hz) }\end{array}$ & $\begin{array}{l}\operatorname{snr} \\
(d B)\end{array}$ & $\begin{array}{l}\text { Occupied frequencies } \\
\qquad(\mathrm{Hz})\end{array}$ & $\begin{array}{c}\text { Elapsed time } \\
(s)\end{array}$ \\
\hline \multirow{12}{*}{ ED } & \multirow{4}{*}{1,000} & -10 & All bands & 97.37 \\
\hline & & 0 & {$[740-1,260]$} & 97.01 \\
\hline & & 10 & {$[740-1,260]$} & 97.91 \\
\hline & & 20 & {$[740-1,260]$} & 98.46 \\
\hline & \multirow{4}{*}{$\begin{array}{l}1,000 ; 1,500 \\
2,000\end{array}$} & -10 & All bands & 97.52 \\
\hline & & 0 & {$[740-1,260],[1,240-1,760],[1,740-2,260]$} & 99.91 \\
\hline & & 10 & {$[740-1,260],[1,240-1,760],[1,740-2,260]$} & 97.37 \\
\hline & & 20 & {$[740-1,260],[1,240-1,760],[1,740-2,260]$} & 96.32 \\
\hline & \multirow{4}{*}{$\begin{array}{c}1,000 ; 1,500 ; \\
2,000 ; 2,500 ; \\
3,000\end{array}$} & -10 & All bands & 99.21 \\
\hline & & 0 & $\begin{array}{c}{[740-1,260],[1,240-1,760],[1,740-2,260],[2,240-} \\
2,760],[2,740-3,260]\end{array}$ & 96.96 \\
\hline & & 10 & $\begin{array}{c}{[740-1,260],[1,240-1,760],[1,740-2,260],[2,240-} \\
2,760],[2,740-3,260]\end{array}$ & 97.68 \\
\hline & & 20 & $\begin{array}{c}{[740-1,260],[1,240-1,760],[1,740-2,260],[2,240-} \\
2,760],[2,740-3,260]\end{array}$ & 98.51 \\
\hline \multirow{12}{*}{$\mathrm{CD}$} & \multirow{4}{*}{$1,000 \mathrm{i}=06$} & -10 & 996 & 106.18 \\
\hline & & 0 & 996 & 106.30 \\
\hline & & 10 & 996 & 107.11 \\
\hline & & 20 & 996 & 110.48 \\
\hline & \multirow{4}{*}{$\begin{array}{l}1,000 ; 1,500 \\
2,000 \mathrm{i}=06\end{array}$} & -10 & $253,996,1,503,1,992,2,011$ & 103.77 \\
\hline & & 0 & $253,996,1,503,1,992$ & 106.40 \\
\hline & & 10 & $253,996,1,503,1,992$ & 106.51 \\
\hline & & 20 & $253,996,1,503,1,992$ & 104.86 \\
\hline & \multirow{4}{*}{$\begin{array}{c}1,000 ; 1,500 ; \\
2,000 ; 2,500 ; \\
3000 \mathrm{i}=06\end{array}$} & -10 & $\begin{array}{c}0,253,507,996,1,503,1,992,2,011,2,500,2,988 \\
3,007\end{array}$ & 107.30 \\
\hline & & 0 & $0,253,507,996,1,503,1,992,2,500,3,007$ & 108.08 \\
\hline & & 10 & $0,253,507,996,1,503,1,992,2,500,3007$ & 107.23 \\
\hline & & 20 & $0,253,507,996,1,503,1,992,2,500,3,007$ & 109.12 \\
\hline
\end{tabular}


Lina María Sepúlveda Cano - Jhon Jair Quiza Montealegre - Camilo Gil Taborda - Jorge Andrés Gómez

\begin{tabular}{|c|c|c|c|c|}
\hline Technique & $\begin{array}{c}\text { Sources } \\
(\mathrm{Hz})\end{array}$ & $\begin{array}{l}s n r \\
(d B)\end{array}$ & $\begin{array}{l}\text { Occupied frequencies } \\
(\mathrm{Hz})\end{array}$ & $\begin{array}{c}\text { Elapsed time } \\
(s)\end{array}$ \\
\hline \multirow{12}{*}{ MS-ICA } & \multirow{4}{*}{1,000} & -10 & $1,000.5$ & 0.30 \\
\hline & & 0 & $1,000.5$ & 0.08 \\
\hline & & 10 & $1,000.5$ & 0.05 \\
\hline & & 20 & $1,000.5$ & 0.07 \\
\hline & \multirow{4}{*}{$\begin{array}{l}1,000,1,500, \\
2,000\end{array}$} & -10 & $1,001,2,002,4,732.2$ & 0.29 \\
\hline & & 0 & $1,001,2,002$ & 0.12 \\
\hline & & 10 & $1,001,2,002$ & 0.15 \\
\hline & & 20 & $1,001,2,002$ & 0.12 \\
\hline & \multirow{4}{*}{$\begin{array}{c}1,000,1,500, \\
2,000,2,500, \\
3,000\end{array}$} & -10 & $1,001.5,2,000.5,2,501.2,3,002$ & 0.13 \\
\hline & & 0 & $1,001.5,1,499.7,2,501.2,3,002$ & 0.10 \\
\hline & & 10 & $1,001.5,1,499.7,2,501.2,3,002$ & 0.09 \\
\hline & & 20 & $1,001.5,2,000.5,3,002$ & 0.38 \\
\hline
\end{tabular}

Source: authors

The proposed MS-ICA technique exhibits a suitable compromise between time and performance. The algorithm is fast enough to sense without causing long interruptions in transmissions. The major disadvantage is the misdetection of some sources. To overcome this inconvenient, it is recommended to run the algorithm a couple of times and increase the amount of information about the frequency occupancy. The remarkable aspect is the elapsed time $(0.15 \pm 0.1 \mathrm{~s})$, especially for online applications with demanding time issues.

\section{CONCLUSION}

A technique for spectrum sensing based on blind source separation is explored. The methodology includes a single-to-multichannel procedure through singular spectrum analysis (SSA), aiming to simulate a MIMO communication system. In this work, three approaches were tested: Energy Detection, Cyclostationary Analysis and the proposed scheme with MS-ICA. The methodology is proved on a collection of artificial mixture signals with different frequencies and noise power. The proposed scheme is able to detect most of the sources, even with high noise presence, with low time consumption. The most accurate technique is Cyclostationary Analysis but it is more demanding in terms of time than the others. As future work, it would be interesting to deal with other kinds of modulations, codifications and channel models. 


\section{ACKNOWLEDGMENTS}

This work is supported by "Validación de algoritmos de aprendizaje automático usados en sistemas de telecomunicaciones de radio cognitiva (Código No. 737)" financed by Universidad de Medellín and by "Ayudas para la realización del doctorado" (RR01/2011) of the Universidad Politécnica de Madrid y TEC2012-38630-C04-01 del Ministerio de Educación de España"

\section{REFERENCES}

1. M. Sarijari, A. Marwanto, N. Fisal, S. K. S. Yusof, R. Rashid \& M. Satria, "Energy detection sensing based on GNU radio and USRP: An analysis study", in Proceedings of the 2009 IEEE 9th Malaysia International Conference on Communications, Kuala Lumpur, Malaysia, Dec. 15-17, 2009, pp. 338-342. Available: https://pdfs.semanticscholar.org/2319/11ca01bab559aed1 7bd745bb6ae4c97a8d70.pdf.

2. Y. Hassan, M. El-Tarhuni \& K. Assaleh, "Learning-based spectrum sensing for cognitive radio systems", Journal of Computer Networks and Communications, vol. 2012, pp. 1-14, 2012. Available: https:/www.hindawi.com/journals/jenc/2012/259824/

3. A. Mate, K. H. Lee \& I. T. Lu, "Spectrum sensing based on time covariance matrix using GNU radio and USRP for cognitive radio", in: 2011 IEEE Long Island Systems, Applications and Technology Conference (LISAT), Farmingdale, NY, USA, May 6, 2011, pp. 1-6.

4. G. Nautiyal \& R. Kumar, "Spectrum sensing in cognitive radio using matlab", International Journal of Engineering and Advanced Technology (IJEAT), vol. 2, no. 5, pp. 529-532, Jun. 2013.

5. Z. Xuping \& P. Jianguo, "Energy-detection based spectrum sensing for cognitive radio", in IET Conference on Wireless, Mobile and Sensor Networks (CCWMSN07), Shangai, China, Dec. 12-14, 2007, pp. 944-947.

6. H. Arslan, Cognitive Radio, Software Defined Radio, and Adaptive Wireless Systems (Signals and Communication Technology). New York: Springer-Verlag, 2007.

7. M. Rahman, A. Haniz, S. Khadka, S., Iswandi, Gahadza, M., Kim, M., ichi Takada, J. "Development of spectrum sensing system with GNU radio and USRP to detect emergency radios", IEICE, The Institute of Electronics, Information and Communication Engineers, Sendai, Japan, Technical Report SR2009-57, Oct. 2009.

8. A. Fehske, J. Gaeddert \& J. Reed, "A new approach to signal classification using spectral correlation and neural networks", in DySPAN 2005. First IEEE International Symposium on New Frontiers in Dynamic Spectrum Access Networks, Baltimore, USA, Nov. 8-11, 2005. pp. 144-150.

9. S. Chaudhari, "Spectrum sensing for cognitive radios: Algorithms, performance, and limitations", Ph. D. thesis, Aalto University, Greater Helsinki, Finland, 2012. 
10. S. Da, G. Xiaoying, C. Hsiao-hwa \& Q. Liang, "Fast cycle frequency domain feature detection for cognitive radio systems", Arxiv, p. 4, Ar. 6, 2009. Available: https://archive.org/details/ arxiv-0903.1183

11. M. Calabro, "A Cooperative Spectrum Sensing Network with Signal Classification Capabilities". Ph. D. thesis, Worcester Polytechnic Institute, Worcester, Massachusetts, 2010.

12. A. G. Ferrer, E.G. Prieto \& D. Peña, "Exploring ICA for time series decomposition”, Working Paper 11-16, Statistics and Econometrics Series 11, May 2011. Available: http://orff.uc3m.es/ bitstream/handle/10016/11285/ws111611.pdf?sequence $=1$

13. L. Molgedey \& H. G. Schuster, "Separation of a mixture of independent signals using time delayed correlations", Physical Review Letters, vol. 72, 3634-3637, 1994.

14. V. Krishnaveni, S. Jayaraman, P. M. Kumar, K. Shivakumar \& K. Ramadoss, "Comparison of independent component analysis algorithms for removal of ocular artifacts from electroencephalogram", Meas. Sci. Rev. J, vol. 5, no. 2, pp. 67-78, 2005.

15. Hongli, Sun, Y.: "The study and test of ICA algorithms", in 2005 Proceedings International Conference on Wireless Communications, Networking and Mobile Computing, vol. 1, Wuhan, China, Sept. 23-26, 2005, pp. 602-605.

16. T. Kolenda, L. K. Hansen \& J. Larsen, "Signal detection using ICA: Application to chat room topic spotting", in $3^{\text {rd }}$ International Conference on Independent Component Analysis and Blind Source Separation, ICA'2001, San Diego, USA, Dec. 9-13, 2001, pp. 540-545. Available: http:// cogsys.imm.dtu.dk/publications/2001/kolenda.ica2001.pdf

17. H. G. Ma, Q. B. Jiang, Z. Q. Liu, G. Liu \& Z. Y. Ma, “A novel blind source separation method for single-channel signal”, Signal Processing, vol. 90, no. 12, pp. 3232-3241, 2010.

18. S. S. Kalamkar \& A. Banerjee, "On the performance of generalized energy detector under noise uncertainty in cognitive radio", in National Conference on Communications (NCC), Delhi, India, Feb. 15-17, 2013. pp. 1-5. 